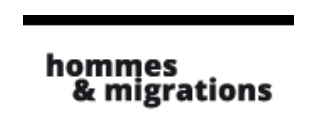

\section{Hommes \& migrations}

Revue française de référence sur les dynamiques

migratoires

$1320 \mid 2018$

Au prisme de la consommation

\title{
Générations de femmes franco-maghrébines
}

La consommation comme marqueur des dynamiques

intergénérationnelles

Ranam Alkayyali et Virginie Silhouette-Dercourt

\section{(2) OpenEdition}

Journals

Édition électronique

URL : http://journals.openedition.org/hommesmigrations/4042

DOI : 10.4000/hommesmigrations.4042

ISSN : 2262-3353

Éditeur

Musée national de l'histoire de l'immigration

Édition imprimée

Date de publication : 1 janvier 2018

Pagination : 21-28

ISBN : 978-2-919040-40-7

ISSN : $1142-852 X$

Référence électronique

Ranam Alkayyali et Virginie Silhouette-Dercourt, «Générations de femmes franco-maghrébines », Hommes \& migrations [En ligne], 1320 | 2018, mis en ligne le 01 janvier 2021, consulté le 06 janvier 2021. URL : http://journals.openedition.org/hommesmigrations/4042 ; DOI : https://doi.org/10.4000/ hommesmigrations.4042 


\title{
GÉNÉRATIONS DE FEMMES FRANCO-MAGHRÉBINES : LA CONSOMMATION COMME MARQUEUR
DES DYNAMIQUES INTERGÉNÉRATIONNELLES
}

Par RANAM ALKAYYALI, chercheuse postdoctorante en sciences de gestion, université Paris-Est Créteil (UPEC), et VIRGINIE SILHOUETTE-DERCOURT, maître de conférences (HDR) en sciences de gestion, université Paris-13, CEPN, Centre Marc-Bloch, Berlin.

\author{
Au sein d'une famille, les choix en matière d'alimentation, \\ d'habillement ou de loisirs représentent autant de vecteurs \\ de transmission ou de contrôle, de jeux de pouvoir et \\ d'émancipation. C'est le cas des pratiques de consommation \\ des jeunes françaises d'origine maghrébine et de leurs mères, \\ qui contribuent à redéfinir les relations familiales. Une enquête \\ fait apparaître les points de rencontre et de tensions entre \\ des imaginaires concurrents au sein de ces familles: \\ celui de la culture des parents et celui de la société dominante. \\ Ainsi, en consommant, les filles et les mères révèlent les échelles \\ de valeurs attachées à leurs pratiques respectives.
}

Du fait de la longue histoire coloniale de la France, l'étude des familles d'origine maghrébine ${ }^{1}$ s'est peu à peu employée à saisir la façon dont s'organisent et évoluent les rapports entre les individus et l'institution familiale, entre la sphère publique et la sphère privée ${ }^{3}$. Dans ces familles comme dans beaucoup d'autres, la migration transforme les relations dans le couple mais également entre les générations ${ }^{4}$. L'enjeu est de réinventer des modèles de vivre-ensemble qui modifient les transmissions et les parcours individuels. Les mères deviennent, par exemple, des médiatrices entre les enfants et leur père, tandis que les jeunes filles cherchent à se frayer leur propre chemin entre discours émancipateurs, respect de la cohésion familiale et marges

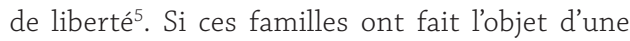
attention croissante, la place et le rôle de la consommation qui s'y déploie restent un angle mort, tandis qu'un certain nombre de pratiques qui leur sont justement attribuées font aujourd'hui débat. La consommation n'est ici pas entendue comme une simple transaction économique mais comme une activité symbolique qui permet de communiquer et d'échanger du sens ${ }^{6}$. 
Adossée à des travaux sur les " écarts d'acculturation » et s'appuyant sur une double enquête qualitative menée sur une plateforme d'entraide professionnelle et dans un lieu de culte musulman ${ }^{7}$, cette recherche tend à montrer qu'à l'intérieur des familles franco-maghrébines la consommation permet de négocier les rapports entre générations. Derrière les choix d'aliments et de produits de beauté, de lieux de restauration et de pratiques de consommation se dessinent des lignes de tension entre les mères et les filles ainsi que des tentatives de dépassement. La consommation les interroge mutuellement. Elle devient une arène dans laquelle se jouent des stratégies de renforcement du lien, de construction de frontières ou de ruptures entre générations de femmes franco-maghrébines.

\section{La famille dans la migration}

Dans l'aventure migratoire, la famille constitue le garant d'une certaine stabilité affective lorsque les assises habituelles de chacun des membres sont mises à rude épreuve et qu'il faut s'adapter à un nouveau quotidien ${ }^{8}$.

On peut, cependant, se demander si la structure familiale n'est pas fragilisée par cette rupture et si les transmissions intergénérationnelles n’en sortent pas profondément remodelées. Le lien entre générations est-il bousculé par un nouveau contexte de vie et de consommation ? Existe-t-il des écarts entre générations concernant le rythme et les contenus d'adaptation ? Comment la famille réagit-elle à la transformation des enfants qui sont des catalyseurs d'acculturation ${ }^{9}$ ? Comment est-ce que ces ajustements s'expriment dans le choix des objets consommés ou des pratiques mises en œuvre?

Ces questionnements ont ouvert un large champ de recherche pour penser le fait familial dans la migration, notamment dans le cas des familles maghrébines, et l'ont éclairé de différentes manières. Deux grands courants se sont ainsi constitués. Le premier, toujours présent et dominant, retient en quelque sorte le point de vue de la société d'accueil, la famille étant alors conceptualisée comme facilitateur ou frein à l'intégration et comme lieu de transmission culturelle. Le deuxième, plus récent, valorise le parcours et l'autonomie du migrant qui, grâce à ses liens familiaux, serait l'acteur d'une mondialisation «par le bas ${ }^{10}$ ». Les recherches appartenant au premier courant s'intéressent plus particulièrement aux relations intergénérationnelles et aux conflits qui seraient à même d'apparaitre du fait de "modèles " familiaux différents : selon cette conception, les familles «traditionnelles » s'organiseraient autour d'un modèle patriarcal, alors que le modèle de famille «moderne » et occidental valoriserait la conquête de l'autonomie, l'égalité des sexes et le partage des tâches ${ }^{11}$. Les enquêtes menées soulignent effectivement la persistance d'une forme de modèle traditionnel dans les familles immigrées qui se traduit soit par des modes de cohabitation particuliers ${ }^{12}$, soit par la transmission de certaines valeurs partagées (décence ou respect patriarcal par exemple) ${ }^{13}$ sans que les conséquences en termes de consommation quotidienne ne soient abordées.

7. Cette double enquête a été réalisée en 2012 sur une plateforme d'entraide professionnelle - Les Dérouilleurs - puis grâce à 19 entretiens semi-directifs menés dans un lieu de culte musulman (à la Mosquée Sahaba, 4, rue Jean Gabin, 94000 Créteil). Les 19 entretiens ont ainsi été menés avec 12 jeunes femmes et 7 mères. Les jeunes femmes originaires d'Algérie (8), du Maroc (3) et de Tunisie (1) avaient entre 20 et 36 ans et étaient étudiantes ou exerçaient une activité professionnelle. Les mères originaires d'Algérie (4), du Maroc (2) ou de Tunisie (1) avaient entre 57 et 63 ans et étaient femmes au foyer (niveau d'étude : baccalauréat). Les mères et les jeunes femmes étaient interrogées sur leur vie en France, sur leurs liens avec leur pays d'origine et avec leur religion, et sur leurs habitudes de consommation. 8. Marie-Rose Moro, " D'où viennent ces enfants si étranges ? Logiques de l'exposition dans la psychopathologie des enfants de migrants ", in Nouvelle revue d'ethnopsychiatrie, $n^{\circ} 12,1989$, pp. 69-84. 9. François-Charles Wolff, Claudine Attias-Donfut, "Les comportements de transferts intergénérationnels en Europe ", in Économie et statistique, vol. 403, $\mathrm{n}^{\circ}$ 1, pp. 117-141. 10. Voir Jocelyne Streiff-Fénard, "Modèles familiaux, transmissions intergénérationnelles et identités ", in Marie Poinsot, Serge Weber (dir.), Migrations et mutations de la société française. L'état des savoirs, Paris, La Découverte, 2014, pp. 240-247. 11. Beate Collet, Emanuelle Santelli, op. cit. ; Vincenzo Cicchelli, " Les jeunes adultes comme objet théorique ", in Recherches et prévisions, vol. 65 , $\mathrm{n}^{\circ}$ 1, pp. 5-18. 12. Claudine Attias-Donfut, François-Charles Wolff, Le destin des enfants d'immigrés. Un désenchaînement des générations, Paris, Stock, 2009. 13. Didier Lapeyronnie, Ghetto urbain. Ségrégation, violence, pauvreté en France aujourd'hui, Paris, Robert Lafont, 2008. 


\section{Les familles maghrébines: entre continuités et ruptures}

Dans le cas des familles maghrébines ${ }^{14}$, les travaux pionniers d'Abdelmalek Sayad ${ }^{15}$ en France ont insisté sur les trajectoires individuelles et sur les conditions sociales de la migration. Si, dans un premier temps, les logiques migratoires étaient marquées par un contexte colonial et par la situation particulière de migrants issus de milieux ruraux, elles se sont complexifiées et diversifiées. Les hommes ne sont plus seuls à migrer, comme durant les vagues migratoires des années 1950 et 1960. À leur tour, soit dans le cadre du regroupement familial, soit depuis les années 1990 pour suivre des études ou à la recherche d'emploi, les femmes transforment les profils des familles migrantes d'origine maghrébine ${ }^{16}$.

La littérature a cependant documenté la permanence de difficultés sociales particulières auxquelles sont confrontées ces familles, que ce soit au niveau des expériences de discrimination ou des processus de relégation spatiale et des difficultés d'accès au marché du travail ${ }^{17}$. Face à ce contexte, les enquêtes soulignent des différences entre générations : les premières générations de migrants maintiennent un lien avec la famille au pays, tandis que les secondes, nées ou socialisées majoritairement en France, bousculent les contours de la famille traditionnelle qui devient «nucléaire $»^{18}$. Les statuts parentaux sont reformulés : la place des pères dans la société d'accueil contribue à bousculer leur prestige, tandis que les mères acquièrent de nouvelles possibilités de négocier entre différents modèles culturels de maternité.

Cependant, ces changements s'accompagnent d'une permanence de modèles plus anciens de domesticité. Les recherches montrent que les enfants socialisés au sein de familles toujours marquées par ces modèles diversifient leurs rapports aux deux sociétés et instaurent une double appartenance ${ }^{19}$ qui peut s'exprimer par des pratiques de consommation. Une visibilité croissante d'offres commerciales (boucherie halal) et de produits associés à des pratiques religieuses témoigne de cette évolution. Le marché du halal est en forte croissance : $59 \%$ des musulmans interrogés affirment consommer systématiquement de la viande halal, et $28 \%$ occasionnellement ${ }^{20}$.

\section{La consommation comme marqueur des processus migratoires}

On assiste en France à l'émergence de recherches qui prennent la consommation comme point d'entrée en vue d'étudier les phénomènes migratoires. Partant de l'hypothèse que les « migrants " n'arrivent pas sans passé ni habitudes de consommation, ces enquêtes s'intéressent aux transformations qui s'opèrent dans leurs modes de consommation et à la façon dont ces derniers reflètent leurs processus d'adaptation. Au départ, ces recherches ne visaient majoritairement qu'une seule génération, sans rendre compte des interactions pouvant se mettre en place entre les deux 
générations ${ }^{21}$ au sein d'une famille. Or, aux ÉtatsUnis, en 1938, l'historien Marcus Lee Hansen s'attachait déjà à décrire les modes de transmissions intergénérationnelles d'une identité culturelle ${ }^{22}$ qui s'expriment par la consommation : certaines générations étant plus enclines que d'autres à revisiter un « héritage culturel » sous une forme symbolique, par exemple autour de pratiques folkloriques, religieuses, ou de façons de se vêtir. Les objets de consommation et la culture matérielle transmis entre les générations familiales

Ce qui est transmis entre les générations peut être

tangible ou intangible, et le canal de transmission varie entre l'observation, l'imitation, ou l'initiation. feraient ainsi partie du mythe familial et en deviendraient les supports de la mémoire ${ }^{23}$.

Pour mettre en scène cette identité familiale, ses membres mobiliseraient différentes ressources symboliques à travers différentes formes de communication et de consommation $^{24}$. Il semblerait que ces ressources jouent un rôle essentiel dans la construction de l'histoire et de l'identité familiale. Ce qui est transmis entre les générations peut être tangible ou intangible, et le canal de transmission varie entre l'observation, l'imitation, ou l'initiation ${ }^{25}$. Ces modes de transmission chers à la théorie de la socialisation supposent un phénomène de passage entre deux parties : un donateur (parent) et un récepteur (enfant). Plusieurs autres facteurs peuvent avoir un impact sur ces transmissions, tels que le cercle familial élargi, l'écart social entre les parents et leurs enfants, la classe sociale, lâge, le genre, et les amis. Ils peuvent être descendants - par exemple, la mère participe à la socialisation de sa fille -, ou ascendants - la fille joue le rôle de modèle pour sa mère. Mais, dans des familles immigrées maghrébines ${ }^{26}$, la question est de savoir comment se mettent en place ces modes de transmission tels qu'ils s'expriment par la consommation, quels en sont les contenus et dans quel sens ils fonctionnent.

\section{Les écarts d'acculturation, porte d'entrée sur la consommation familiale}

Un des corpus fréquemment mobilisés pour rendre compte de cette consommation familiale s'organise autour de la notion d' "écart d'acculturation ${ }^{27}$ ". Issue des travaux menés en psychologie interculturelle sur le bien-être des descendants d'immigrés, cette notion part du constat que, finalement, l'enjeu majeur des familles immigrées est la différence vécue entre les générations en termes d'adaptation au pays d'accuei ${ }^{28}$. Les décalages peuvent concerner les rythmes ou les contenus d'adaptation d'une génération par rapport à l'autre.

Dans certains pays, on observe une adaptation rapide des descendants d'immigrés à leur nouvel environnement dans lequel ils sont très souvent scolarisés, en comparaison de leurs parents restés

21. Voir, par exemple, Virginie Silhouette-Dercourt, Beauté ethnique sous tension, entre marginalisation, injonctions républicaines et inventivité du quotidien, Caen, éd. EMS, 2017 ; "Consommer comme ici ou comme là-bas? Les dimensions identitaires de la consommation de cosmétiques des jeunes femmes issues de l'immigration subsaharienne en France ", in Hommes \& Migrations, $n^{\circ}$ 1286-1287, 2010, pp. 190-198; Andrew Lindridge, "Acculturation, religion, and consumption in normative political ideology ", in Ann L. McGill, Sharon Shavitt (dir.), Advances in Consumer Research, vol. 36, Duluth, MN : Association of Consumer Research, 20og; Nil Ozcaglar-Toulouse, Tuba Üstuner, "How do historical relationships between the host and home countries shape the immigrants' consumer acculturation processes? ", in Advances in Consumer Research, vol. 36, 2009. 22. Hansen, résumait cette loi de la sorte: "ce que le fils veut oublier, le petit-fils souhaite se rappeler.", 1938. Voir Hansen Marcus Lee, The Problem of the third Generation Immigrant, Rock Island, Augusta Historical Society, 1938. Ce discours a été réimprimé avec une préface d'Oscar Handlin, in Commentary, vol. 14, $\mathrm{n}^{\circ}$ 5, 1952, pp. 492-500. 23. Serge Tisseron, Comment l'esprit vient aux objets, Paris, PUF, 2016. 24. Amber Epp, Linda Price, "Family Identity: A framework of identity interplay in consumption practices ", in Journal of Consumer Research, vol. 35, $n^{\circ}$ 1, $2008, \mathrm{pp} .50-70$. 25. Elizabeth Moore, William Wilkie, Julie Alder, "Lightening the torch: How do intergenerational influences develop? ", in Advances in Consumer Research, vol. 28, $n^{\circ}$ 1, pp. 287-293. 26. Voir l'enquête suivante qui s'intéresse à ces questions mais du point de vue du bien-être psychologique des jeunes descendants d'immigrés en Europe : John W. Berry, Jean S. Phinney, David L. Sam, Paul Vedder, "Immigrant youth: Acculturation, identity, and adaptation", in Applied Psychology, vol. 55, n 3, pp. 303-322. 27. Pour rappel, l'« acculturation " est un concept issu de l'anthropologie qui désigne "l'ensemble des changements culturels résultant des contacts continus et directs, entre deux groupes culturels indépendants". Voir Robert Redfield, Ralph Linton, Melville J. Herkovits, "Memorandum for the study of acculturation ", in American Anthropologist, vol. 38, n¹, 1936, pp. 149-152. 28. Anna S. Lau, Kristen M. McCabe, May Yeh M, Ann. F. Garland, Patricia A. Wood, Richard L. Hough, "The acculturation gap-distress hypothesis among high-risk mexican american families ", in J Fam Psychol, vol. 19, $n^{\circ}$ 3, pp. 367-375. 
attachés aux valeurs du pays d'origine telles qu'ils se les imaginent ${ }^{29}$. Parfois, cependant, se produit un phénomène contraire: ce sont les enfants qui restent plus attachés aux valeurs qu'ils associent au pays d'origine de leurs parents ${ }^{30}$. Mais, dans un cas comme dans l'autre, il se produit des décalages entre générations, parfois source de tensions. Ainsi, différentes études montrent que les décalages vécus entre les mères et leurs filles peuvent entraîner des phénomènes de souffrance psychologique, tels que l'anxiété ou la dépression chez les filles ${ }^{31}$. De la même manière, dans le cas des parents, la préférence de leurs enfants pour des valeurs associées à leur pays d'origine altère la relation intergénérationnelle : les enfants deviennent moins favorables aux pratiques parentales, tandis que les parents deviennent moins susceptibles de surveiller le comportement de leurs enfants ${ }^{32}$. Il est ainsi possible de distinguer entre deux écarts: l'écart d'acculturation, d'une part, et l'écart d'enculturation, d'autre part ${ }^{33}$. Le premier représenterait la différence entre la stratégie d'adaptation de la mère et celle de sa fille vis-à-vis du pays d'accueil, alors que le deuxième représente la différence entre la stratégie d'adaptation de la mère et celle de sa fille vis-à-vis du pays d’origine.

\section{Un moyen de dialoguer entre générations}

Pour de nombreuses mères et jeunes femmes interrogées, la consommation est utilisée pour atténuer les écarts qui pourraient apparaître entre générations.

Par exemple, les mères maghrébines décident de rejoindre leurs filles dans leurs lieux de consommation comme les cinémas, les cafés, et les restaurants ${ }^{34}$. Voici l'exemple de Mahya : «Je sors avec mes filles et on va au cinéma. Une fois par mois, je sors avec mes filles et on mange ensemble. Comme elles savent mieux que moi, ce sont elles qui choisissent et moi je suis. Elles me disent: "Mahya, il y a un nouveau resto", et voilà, on sort. Une fois, nous sommes parties dans un resto pakistanais, une autre fois, indien, ou au kebab chez les Turcs. Elles aiment bien McDonald's, mais je n'aime pas le pain de McDonald's, je ne suis pas trop McDonald's, je suis plutôt restaurant "arabe". Mais je ne dis pas non à mes filles, donc j’y vais. " (Mahya, 61 ans, mère de famille, née en Algérie et arrivée en France à 21 ans.)

Les jeunes femmes à leur tour disent mobiliser la consommation pour construire des passerelles avec leurs mères : elles leur apprennent l'usage d'outils technologiques répandus en France comme les téléphones mobiles et les tablettes, l'achat sur Internet, ou les amènent au centre de loisirs français pour les rapprocher de la « culture française » comme dans le cas de Rabia : « J'ai pris de ma mère la convivialité et elle me De la même manière, dans le cas des parents, la préférence de leurs enfants pour des valeurs associées à leur pays d'origine altère la relation intergénérationnelle: les enfants deviennent moins favorables aux pratiques parentales, tandis que les parents deviennent moins susceptibles de surveiller le comportement de leurs enfants. rappelle souvent mon identité et qui je suis et donc ça c'est important également... Moi, ce que je lui ai transmis dernièrement par exemple, je l'ai amené au cinéma. C'est la première fois de ma vie que je l'amène au cinéma et ça lui a beaucoup plu... C'était un film sur un immigré algérien français. Ce que j'ai envie de lui transmettre, c'est de venir avec moi dans un café prendre un verre. C'est mieux que de rester à la maison, et elle accepte de venir. Avec moi, elle est ouverte. On fait souvent la commande de pizza sur Internet aussi. » (Rabia, 26 ans, étudiante, née en France de parents de nationalité algérienne.)

29. Mayra Bamaca-Colbert, Jochebed Gayles, « Variable-centered and person-centered approaches to studying Mexican-origin mother-daughter cultural orientation dissonance ", in Journal of Youth and Adolescence, vol. 39, n 11, 2010, pp. 1274-1282 ; Dina Birman, Meredith Poff, "Intergenerational differences in acculturation », in Richard E. Tremblay, Michel Boivin, Ray RDeV Peters (dir.), Encyclopedia on Early Childhood Development, Centre of Excellence for Early Childhood Development, Montréal, 2011. 30. Costigan Catherine, Dokis Daphné, "Similarities and differences in acculturation among mothers, fathers, and children in immigrant chinese families", in Journal of Cross-Cultural Psychology, vol. 37, $n^{\circ}$ 6. 31. Ibid., p. 20. 32. Ibid., p. 21. 33. May Kim, Irene Park, "Testing the moderating effect of parent-adolescent communication on the acculturation gap-distress relation in korean american families ", in Journal of Youth and Adolescence, vol. 40, $\mathrm{n}^{\circ}$ 12, 2011, pp. 1661-1673. 34. Contrairement à ce qu'elles vivent parfois au pays où la socialisation des mères se passe alors davantage à l'intérieur de la maison : les femmes retrouvent leurs amies et voisines pendant des visites à domicile. 


\section{Un espace d'aménagement des écarts entre générations}

Dans ce type de dynamique, les jeunes femmes et leurs mères coexistent en respectant les convictions et les façons de vivre de chacune. Cependant, les choix de consommation révèlent des écarts entre générations comme dans l'exemple de Nadine: "Moi, je fume, je ne mange pas halal. J'aime être coquette, en short par exemple. Je mange des fois halal parce que mes enfants ne mangent que halal. Quand ils font ramadan, je leur prépare à manger tous les repas algériens typiques, c'est merveille chez nous, mais voilà chacun a sa vie. » (Nadine, 63 ans, assistante sociale divorcée, née en Algérie et arrivée en France à 16 ans.)

La consommation permet de maintenir le lien, d’aménager deux espaces générationnels qui ne se comprennent pas toujours mais s'acceptent dans leurs particularités.
Des décalages sont constatés entre des jeunes femmes qui émettent des critiques envers la société de consommation «ultramoderne » dans laquelle elles ont été élevées et leurs mères maghrébines portées par ce même modèle de consomma-

tion. Par exemple, Alaa critique les choix vestimentaires de sa mère : "Ma mère s'habille à l'occidentale, jeans et mini-jupes... Des fois, elle met la gandoura ${ }^{35} \grave{a}$ la maison comme toutes les Algériennes, et toujours en cheveux blonds, je ne sais pas pourquoi? "(Alaa, 34 ans, cadre pharmaceutique, née en France de nationalité algérienne.) La consommation de ces jeunes filles est utilisée à la fois pour se différencier de leurs mères, mais aussi pour articuler une forme de métissage et se créer leur propre monde au sein de l'univers familial' ${ }^{36}$.

Hiba, par exemple, est une étudiante francoalgérienne qui respecte le halal dans sa consommation pour se distinguer de la cuisine grasse de sa mère maghrébine : «Mes parents font les courses pour toute la maison. Des fois, je vais toute seule faire mes courses, parce que je n'aime pas ce que ma mère prépare. Elle prépare des repas que personne ne va manger,

comme les soupes et les repas arabes. Moi, je suis habituée à manger sain, des légumes et des fruits, donc je cuisine pour moi du riz, du poisson, ma mère cuisine pour cuisiner pas pour manger sain. » (Hiba, 21 ans, étudiante, née en France de parents de nationalité algérienne.)

Ces jeunes femmes critiquent le style de vie de leurs mères sans imposer le leur à toute la famille. La consommation permet de maintenir le lien, d'aménager deux espaces générationnels qui ne se comprennent pas toujours mais s'acceptent dans leurs particularités.

\section{Un moyen pour les mères de contrôler leurs filles}

Les mères interrogées se présentent comme les garantes d'une forme d'attachement au pays. Elles ont tenté de le transmettre à leurs filles qui, aujourd'hui, vivent souvent à distance d'elles. Voici l'exemple de Jamila : "Le lien avec l'Algérie est très fort. Je vais là-bas chaque année. On essaie de faire ça surtout pour nos enfants. On est dans une autre culture ici et on est arrivés matures et solides pas comme nos enfants... » (Jamila, 57 ans, mère de famille, née en Algérie et arrivée en France à 29 ans.)

La volonté de transmettre un attachement au pays se manifeste, par exemple, par la préparation des repas. "Je cuisine beaucoup, spécialement les repas marocains. Je cuisine très bien marocain et n’importe quel repas: le tagine, c'est le repas préféré pour mon fils qui est né ici. Je prépare aussi pour mes enfants des pieds de veau avec des pois chiches ou le couscous sucré salé. Ils sont nés ici, mais je les ai habitués à manger marocain, ça leur rappelle l’origine de leurs parents. " (Mahjouba, 58 ans, assistante maternelle, née au Maroc et arrivée en France à 21 ans.)

Parfois, ces transmissions sont pourtant source de tensions entre les mères et leurs filles : les jeunes femmes les rejettent et tentent alors de 
s'émanciper. La consommation de produits non halal ou de coloration de cheveux, les voyages sans l'accord des parents, les sorties dans les clubs et discothèques sont autant de comportements mobilisés pour maintenir à distance cette emprise. L'exemple de Lina, qui a décidé de quitter sa famille pour vivre seule à Paris, est révélateur de ces comportements : "Quand jétais au collège, ma mère m'interdisait plein de choses: sortir, colorer mes cheveux... Mon frère devait m'accompagner au collège, je n'avais pas de vie, je n'avais que des interdictions, j'étais triste. Donc, quand je suis arrivée au lycée, je voulais réclamer mes droits et ma vie de tous les jours. Tout cela à cause de ma mère et de son style de vie. Donc je faisais ce que je voulais après l'école, je sortais, je rencontrais les gens, et je me disais : "Voilà, c'est ça la vie." Puis j'ai quitté Rouen, je suis venue à Paris et j’ai eu ma liberté. » (Lina, 29 ans, employée, née en France de parents de nationalité algérienne.)

Dans des cas moins extrêmes, les jeunes femmes adoptent une stratégie de contournement : elles respectent certains codes, par exemple vestimentaires, devant leurs parents mais reprennent un style qu'elles qualifient de "rebelle » à l'extérieur: "Comme je suis consultante, je dois m'habiller un petit peu classique. Mais quand on fait une collecte de charité chez mes parents, on met le "Pakistanais"; un ensemble long qui va jusqu'aux genoux avec pantalon. Pour ma vie de tous les jours, ça va être français. Des fois, j'ai envie de mettre une minijupe à Paris. Mais pour aller au marché avec ma famille, je mets des tuniques des fois par respect, on essaie de ne pas heurter. " (Lana, 34 ans, consultante en entreprise, née en France de parents de nationalité marocaine.)

Dans d'autres situations, les jeunes femmes tentent de construire un dialogue autour de certaines pratiques comme Lana: "Pour le maquillage, ma mère ne met pas de fond de teint, elle utilise le khöl ${ }^{37}$ et le siwak $^{38}$. Une fois, je lui ai acheté une boîte de maquillage à 70 euros et elle ne l'a pas acceptée. Elle a dit qu'elle n'avait pas le temps! Je lui ai dit: "Mais comment tu n'as pas de temps et tu es tout le temps à la maison?" Elle fait toujours comme ça avec moi. Je l'appelle au téléphone, elle dit qu'elle n'a pas de temps, et puis elle raccroche! Elle est occupée par la maison. "

On le voit ici, la volonté des mères de transmettre une forme d'attachement à leur pays d'origine par la consommation, suscite en retour de multiples stratégies des jeunes femmes qui cherchent à la contourner ou à la désactiver.

\section{Un moyen pour les filles de prendre le contrôle}

Dans certains cas, des jeunes femmes sont dans des dynamiques de contrôle. Elles réinterprètent les valeurs religieuses de façon rigoriste, comme Rahaf : "Quand je portais mon voile, cétait pour pratiquer ma religion comme il faut. Mes parents ne m'ont jamais obligé à le porter. Ma mère le portait, mais pour les traditions, et elle ne voulait pas que je le porte parce que je suis encore étudiante et que je n'ai pas encore travaillé. Mais, petit à petit, elle l'a accepté. (...) La différence entre elle et moi, c'est qu'elle porte la djellaba traditionnelle marocaine. C'est normal, c'est son pays d'origine, donc elle aime tout ce qui est coloré, brodé, etc., moi non, je préfère les choses plus simples comme Al Abaya ${ }^{39}$. » (Rahaf, 21 ans, employée en entreprise de télémarketing, née en France de parents de nationalité marocaine.) Dans cet exemple, il s'agit pour Rahaf de négocier sa position vis-à-vis de sa mère. Elle tente ainsi en quelque sorte de contrôler la consommation de toute la famille en imposant sa vision de ce qui serait attendu en termes de pratique religieuse. Les modes de transmission intergénérationnelle sont ici de types ascendants : les filles tentent d'affirmer leur style de vie dans la maison. Ce qui, dans certains cas, peut être source de tensions entre générations. Les rôles maternels semblent alors inversés : les mères sont soumises aux préférences de leurs jeunes filles qui cherchent à imposer des normes de comportement passant par la consommation. 


\section{Conclusion}

La consommation peut être un marqueur des dynamiques intergénérationnelles entre les mères maghrébines et leurs filles en France. Comme dans toute famille, chaque génération se construit en rapport (d'opposition mais aussi parfois en symbiose) avec la génération précédente. Ici, cependant, apparaissent d'autres enjeux de la transmission. Les histoires de consommation mêlées aux histoires de vie révèlent les écarts d'acculturation. En fait, les jeunes femmes francomaghrébines et leurs mères mobilisent la consommation pour articuler et mettre en scène leurs appartenances à la société française mais aussi à leurs familles. En conséquence, on constate la présence de multiples formes d'arrangements dans les discours, car chaque génération vit une forme d'acculturation différente, avec des récits de consommation toujours présents. Certaines jeunes femmes ont rompu les relations avec leurs mères, ce qui reflète les tensions intergénérationnelles qu'elles peuvent parfois vivre. Cependant, pour la majorité de ces mères et de ces jeunes femmes, les processus sont éminemment mouvants et dynamiques. Entre vivre ici et ne pas trahir le lien familial, le parcours est parfois semé d'embûches. La famille offre un espace, un lieu d'expérimentations mais aussi de conflits et de ruptures. La consommation qui s'y déploie reflète ces multiples façons de négocier les rapports entre générations. 